\title{
Diseño y fabricación de máquina para optimizar los procesos de desgrane y selección de granos de maíz
}

\author{
Design and manufacture of machine to optimize \\ the processes of shelling and selection of corn grains
}

\author{
'Flores Ramos, O.P.; Morales Santivañez, W.V.; León, D.; Rebollar, M. \\ Facultad de Ingeniería Mecánica, Universidad Nacional del Centro del Perú \\ Email: opflores@uncp.edu.pe
}

\section{Resumen}

El trabajo está orientado al diseño y fabricación de desgranadora - seleccionadora de maíz para optimizar de dicho proceso, siendo del tipo tecnológico y nivel aplicativo. Tiene como objetivo el diseñar y construir una máquina para el proceso de desgranado de maíz seco y, a la vez, seleccionarlo de acuerdo a dos tamaños de grano, con una capacidad de $150 \mathrm{~kg} / \mathrm{h}$.

Las partes de la máquina, fueron diseñadas y construidas de acuerdo a los manuales de diseño, predominando el acero A-36, de poco peso lo cual facilita su transporte, el sistema formado por un alimentador manual, desgranado por fricción, accionado con fuerza humana, con zaranda para la selección de los granos y recepción en cubetas.

Se realizaron pruebas con diferentes variedades de maíz y de diferentes porcentajes de humedad, obteniendo el correcto desgrane en cualquiera de estas condiciones; por lo que, se concluye que sus partes funcionan de acuerdo al propósito de diseño. Se logró obtener una máquina de desgrane limpio y grano seleccionado sin desperdicios, obteniéndose maíz listo para comercializar.

El aporte social, radica en que esta máquina está al servicio de toda una comunidad rural y su diseño ha mejorado tecnológicamente respecto a las máquinas existentes en el mercado, no lleva motor eléctrico ni de combustión, al actúar con fuerza humana, accionado por una manivela.

Palabras Clave: calidad de la energía, armónico de tensión, armónico de corriente

\begin{abstract}
The work is oriented to the design and manufacture of a corn shaker-sorter for the processing of the aformentioned processes, its type is technological and its level is application. It has as objective designing and building a machine for the dry corn shelling process and at the same time selecting according to two grain sizes, with a capacity of $150 \mathrm{~kg} / \mathrm{h}$.

The machine parts, were designed and constructed according to the design manuals, using A-36 steel predominantly, with low weight which facilitates its transport. The system is formed by a manual feeder, friction shelling, operated by human force, with zaranda for grain selection and bucket reception in

Tests were carried out with different corn varieties and different moisture percentages, obtaining the correct shelling in any of these conditions, so the conclusion is that its parts work according to the design purpose. It was obtained a clean shelling and selected grain machine without waste, obtaining corn ready to be marketed.

The social contribution lies in the fact that this machine serves a whole rural community and its design has improved technologically in relation to existing machines in the market, it does not have an electric or combustion engine, since it acts through human force, operated by a crank.
\end{abstract}

Keywords: power quality, voltage harmonic, current harmonic 


\section{Introducción}

El valle del Mantaro, es un gran productor de maíz, pero existe el problema de desgranar y clasificar el maíz, al hacerse en forma manual, convirtiéndose con ello en una labor tediosa en la clasificación y cansada en el proceso de desgrane, porque se hace manualmente y de mazorca en mazorca, lo que lleva largo tiempo el proceso; además, el maíz desgranado y clasificado, varía su valor en el mercado porque de acuerdo a su tamaño aumenta su valor adquisitivo al momento de venderlo.

Debido a ello, se propuso solucionar dicho problema diseñando y construyendo una máquina desgranadora - clasificadora de granos de maíz para optimizar dichos procesos para los pobladores del sector rural. Al estar orientado al sector rural, esta máquina no lleva motor eléctrico, ni motor de combustión, solo funcionará con energía humana, haciendo uso de mecanismos mecánicos, accionada manualmente por una sola persona, con una capacidad aproximada de $150 \mathrm{~kg} / \mathrm{h}$, si es que es accionada por una persona adulta.

Inicialmente, se realizó el marco teórico del maíz, tales como características y formas de desgranado; así como, los tipos de máquinas existentes en el mercado. Luego se describe brevemente, el método utilizado y se elabora los conceptos de solución. Se plantea también cuatro alternativas de diseño de desgranadoras. Se escoge la alternativa más conveniente que cumpla con todos los requerimientos.

Se procede al diseño mecánico de la máquina considerando fuerzas, velocidades, potencias, para dimensionar y adecuar cada componente, teniendo en cuenta los factores externos para su construcción. Se elabora el plano general de la máquina y de los elementos de la desgranadora seleccionadora de maíz especificando sus dimensiones y tolerancias.

La máquina, es de fácil manejo y fabricado en talleres artesanales de la localidad con materiales de uso común en cerrajería (acero A 36), fáciles de conseguir en el mercado local y a bajo costo.

\section{Materiales y métodos}

\section{Materiales y equipos a utilizar}

Se utilizó el método discursivo con matriz morfológica (Norma VDI 2221), en el cual se puede apreciar las cuatro fases del diseño.

- Fase 1: Planeamiento

- Fase 2: Concepción de la solución

- Fase 3: Elaboración del proyecto

- Fase 4: Elaboración de detalles
Figura 1

Método discursivo

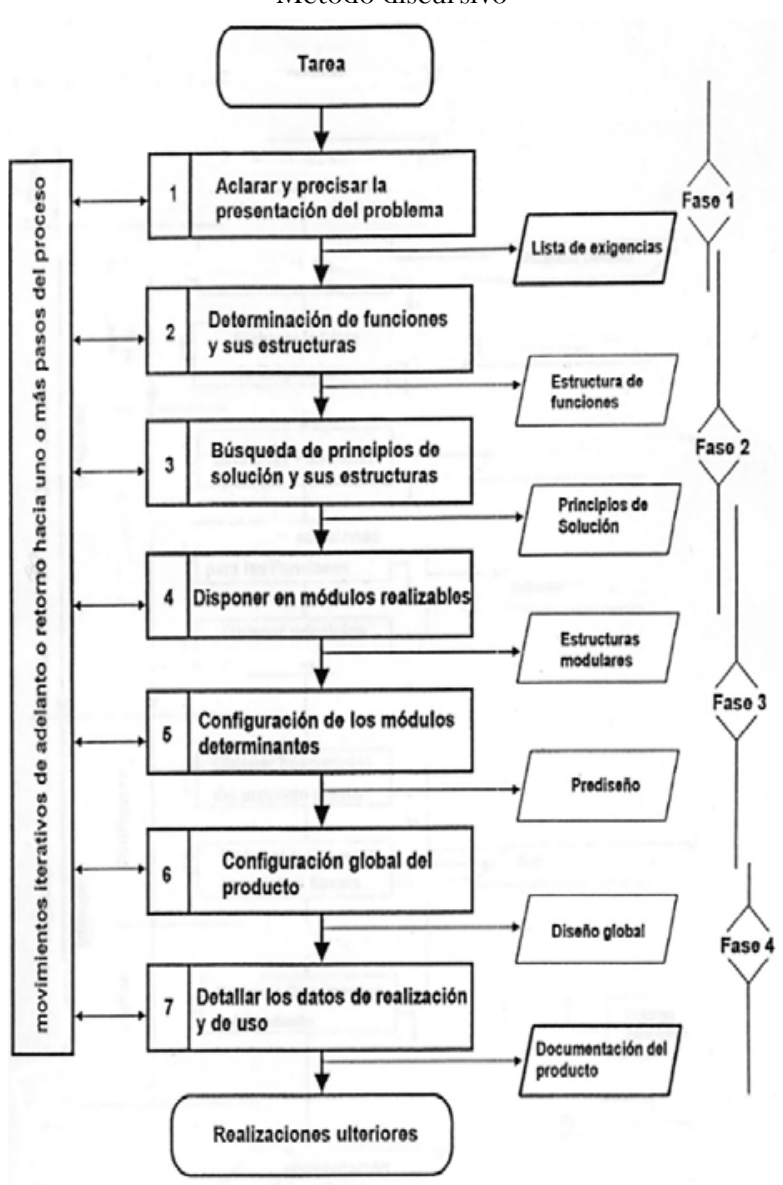

Fuente: VDI 2221

\section{Resultados}

Se realizó una evaluación cualitativa de los conceptos solución y se determinó la solución óptima.

\section{Concepto solución óptimo}

\section{Valor técnico}

Criterio 1 (C1): Fácil fabricación

Criterio 2 (C2): Fácil de transportar

Criterio 3 (C3): Fácil operación

Criterio 4 (C4): Consumo de energía

Criterio 5 (C5): Frecuencia de mantenimiento

Criterio 6 (C6): Nivel de ruido

Los criterios están valorizados entre 1 como mínimos y 5 como máximo.

Valor técnico $=$ suma ponderada / Puntaje máximo

\section{Valor económico}

Costo admisible de fabricación $=\$ 450$

Costo ideal $=0.7 \times$ Costo admisible $=\$ 315$

Valor económico $=$ Costo ideal/Precio aproximado 
Tabla 1

Matriz de decisión técnica

\begin{tabular}{|c|c|c|c|c|c|c|c|c|c|c|}
\hline \multicolumn{2}{|c|}{ Criterios } & $\mathrm{C} 1$ & $\mathrm{C} 2$ & $\mathrm{C} 3$ & $\mathrm{C} 4$ & C5 & C6 & \multirow{2}{*}{$\begin{array}{c}\text { Suma } \\
\text { ponderada }\end{array}$} & \multirow{2}{*}{$\begin{array}{l}\text { Puntaje } \\
\text { máximo }\end{array}$} & \multirow{2}{*}{$\begin{array}{c}\text { Valor } \\
\text { técnico }\end{array}$} \\
\hline & Peso & 0.25 & 0.25 & 0.15 & 0.15 & 0.05 & 0.15 & & & \\
\hline \multirow{2}{*}{ CS1 } & Valor & 4 & 3 & 4 & 4 & 3 & 2 & \multirow{2}{*}{$3.4 \mathrm{O}$} & \multirow{2}{*}{5} & \multirow{2}{*}{$68 \%$} \\
\hline & Puntaje & 1 & 0.75 & 0.50 & 0.50 & 0.15 & 0.30 & & & \\
\hline \multirow{2}{*}{$\mathrm{CS} 2$} & Valor & 3 & 1 & 2 & 3 & 3 & 4 & \multirow{2}{*}{$2.5 \mathrm{O}$} & \multirow{2}{*}{5} & \multirow{2}{*}{$50 \%$} \\
\hline & Puntaje & 0.75 & 0.25 & 0.30 & 0.45 & 0.15 & 0.60 & & & \\
\hline \multirow{2}{*}{ CS3 } & Valor & 4 & 4 & 4 & 4 & 3 & 4 & \multirow{2}{*}{3.95} & \multirow{2}{*}{5} & \multirow{2}{*}{$79 \%$} \\
\hline & Puntaje & 1.00 & 1.00 & 0.60 & 0.60 & 0.15 & 0.60 & & & \\
\hline \multirow{2}{*}{ CS4 } & Valor & 4 & 4 & 4 & 4 & 4 & 2 & \multirow{2}{*}{3.70} & \multirow{2}{*}{5} & \multirow{2}{*}{$74 \%$} \\
\hline & Puntaje & 1.00 & 1.00 & 0.60 & 0.60 & 0.20 & 0.30 & & & \\
\hline
\end{tabular}

Tabla 2

Matriz de decisión económica

\begin{tabular}{lcccc}
\hline Concepto solución & CS 1 & CS 2 & CS 3 & CS 4 \\
\hline Precio aproximado & 380 & 420 & 380 & 450 \\
Valor económico & $83 \%$ & $75 \%$ & $83 \%$ & $70 \%$ \\
\hline
\end{tabular}

\section{Evaluación técnica económica}

Figura 2

Evaluación técnica económica

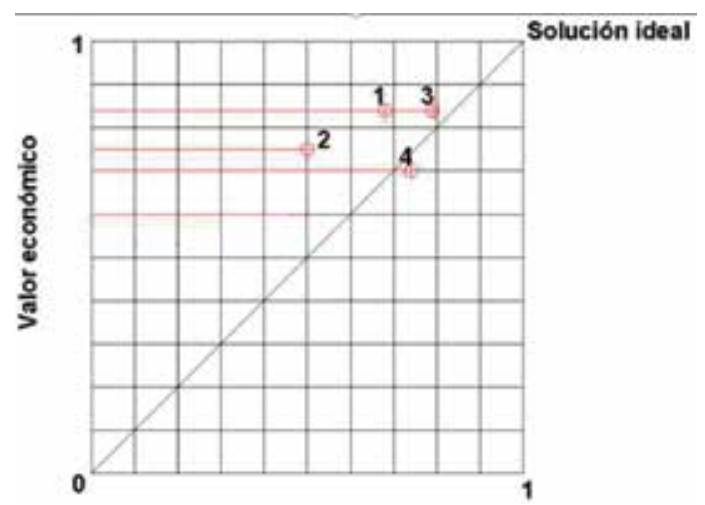

Como el Concepto / solución 3, se acerca más al ideal, se consideró como la solucion óptima.

\section{Diseño de la máquina}

Como ya se identificó como solución óptima, el concepto 3, el cual consta de una alimentación manual, desgranado por fricción, accionado con fuerza humana, con zaranda para la selección de los granos y recepción en cubetas, procedemos a detallarlos:

\section{Selección de la fuente de energía}

La energía para el accionamiento de la máquina debía ser la disponible en una zona rural, estas fuentes de energía son dos: La humana y la animal. Para este caso se eligió la energía humana.

\section{Mecanismo desgranador de maíz}

Fue diseñada por función; es decir, no se realizaron cálculos de esfuerzos ni deformaciones, ya que el mecanismo no sufre deformaciones y los esfuerzos considerables al desgranar el maíz.

Su fabricación fue de fundición, consta de un disco granulado giratorio con entrada cónica y una manivela que hace girar al disco.

Figura 3

Mecanismo desgranador de maíz
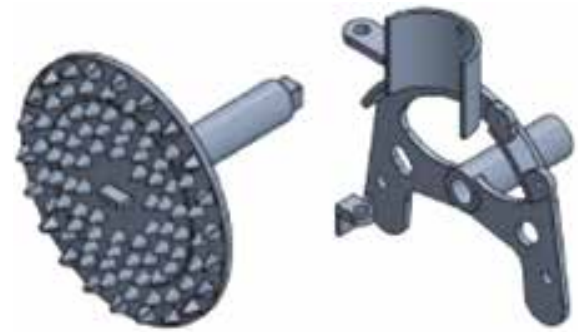

\section{Zaranda seleccionadora}

Este mecanismo consiste en dos plataformas agujereadas con diámetros diferentes para la selección de los granos de maíz, accionados por un eje que tiene un eje excéntrico que hará que la zaranda vibre, sobre un canal de guía horizontal hará que un extremo se desplace en forma horizontal, el alimentador será la manivela accionada por un hombre.

Figura 4

Zaranda seleccionadora de maíz

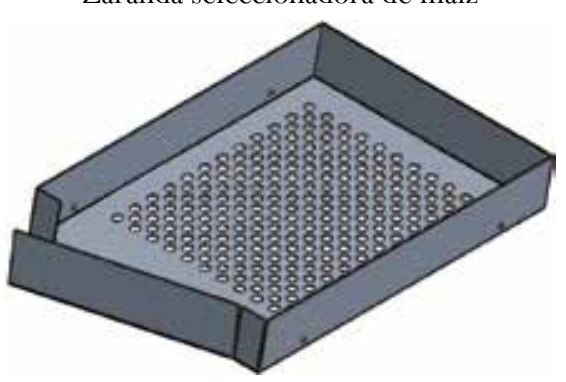


Esta configuración permitió movimientos vibratorios armónicos de la zaranda, el cual favorece para la selección de los granos, pues las vibraciones suaves durante el funcionamiento, permite transportar casi horizontalmente los granos de maíz hacia la salida.

\section{Bastidor de la máquina}

Fabricada de acero A 36, ser analizó los esfuerzos y las deformaciones producidos por las cargas dinámicas, las cuales fueron mínimas dentro de lo permisible.

Figura 5

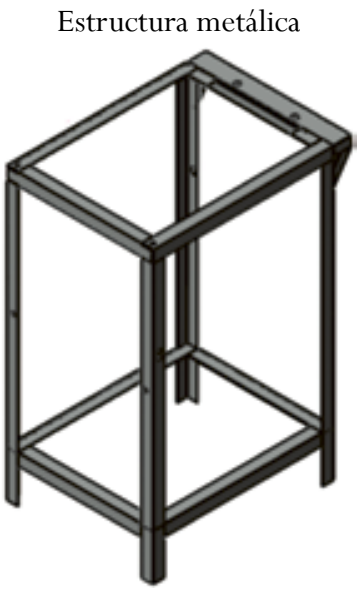

\section{Producto final}

Se presenta la máquina desgranadora seleccionadora de maíz.

\section{Figura 6}

Máquina desgranadora seleccionadora de maíz

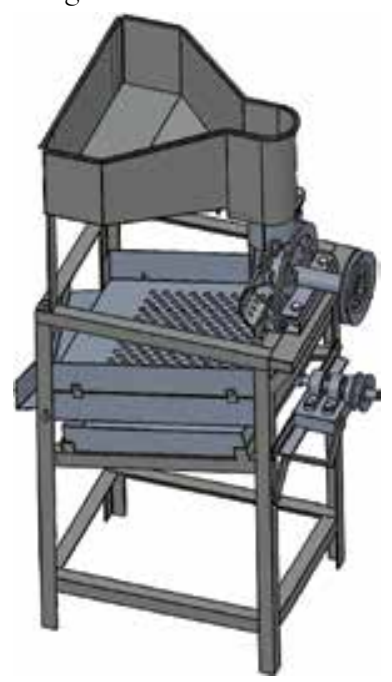

\section{Discusión}

\section{Concepto solución 1}

Este concepto consta de alimentación manual accionado por motor eléctrico, desgranado por impacto o golpe de cadena, con cilindros concéntricos para la selección de los granos y recepción en cubetas.
Figura 7

Concepto solución 1

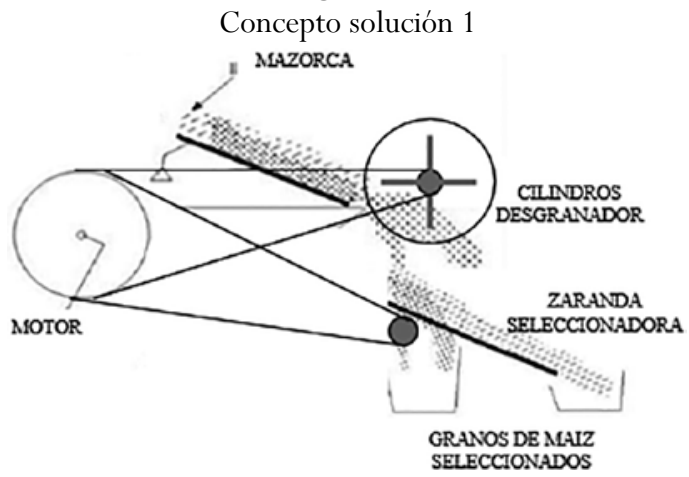

\section{Concepto solución 2}

Este concepto consta de alimentación manual accionado por motor eléctrico, desgranado por impacto o golpe de cadena, con zaranda para la selección de los granos y recepción en cubetas.

Figura 8

Concepto solución 2

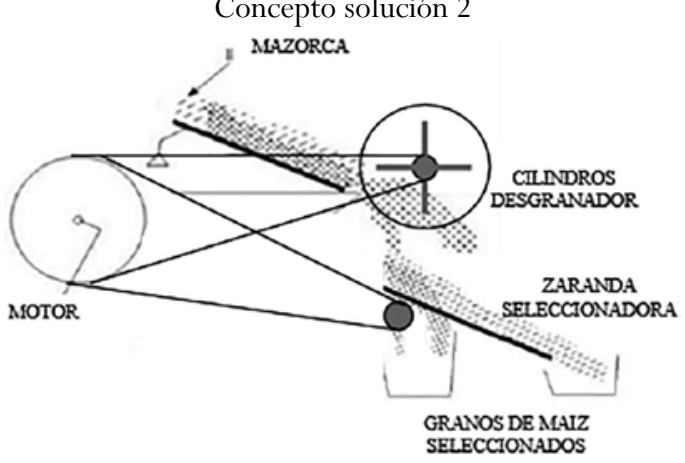

\section{Concepto solución 3}

Este concepto consta de alimentación manual, desgranado por fricción, accionado con fuerza humana, con zaranda para la selección de los granos y recepción en cubetas.

Figura 9

Concepto solución 3

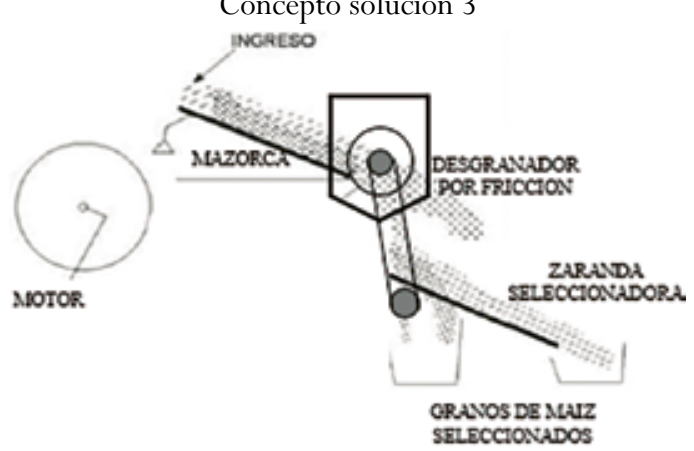

\section{Concepto solución 4}

Este concepto consta de alimentación manual, desgranado por fricción, accionado con fuerza humana, con cilindro concéntrico para la selección de los granos y recepción en cubetas. 
Figura 10

Concepto solución 4

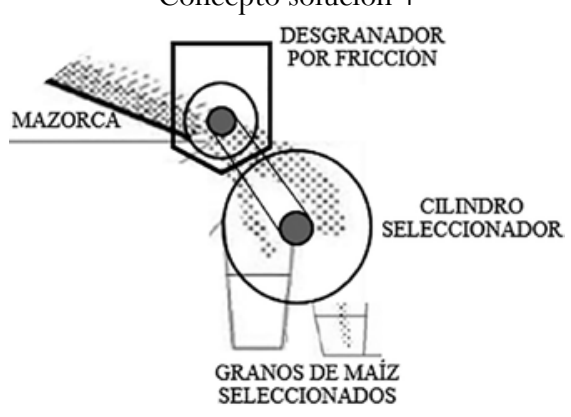

\section{Conclusiones}

- Como se pudo apreciar en la Tabla 1, en la matriz de decisión técnica, el que tiene mejores características técnicas, es el concepto solución 3, que obtiene un valor técnico del $79 \%$, esta solución es alimentación manual, desgranado por fricción, accionado con fuerza humana, con zaranda para la selección de los granos y recepción en cubetas. Mientras que el concepto solución 2, solo obtiene $50 \%$, esta solución es alimentación manual accionado por motor eléctrico, desgranado por impacto o golpe de cadena, con zaranda para la selección de los granos y recepción en cubetas, el golpe es lo que baja el puntaje, debido a que algunos granos de maíz son partidos.

- En cuanto al aspecto económico, según la Tabla 2, en la matriz de solución económica, el concepto solución 1 y concepto solución 3, obtienen $83 \%$, mientras que el concepto solución 4 , que obtiene un valor económico de $70 \%$.

- Mientras que en la tabla de decisión técnica económica la solución óptima es el concepto 3, la cual se acerca más a la solución ideal, como se puede ver en la Figura 2.

\section{Referencias bibliográficas}

Agicolas, C. P. (2000). Perfeccionadora en espiga desgranadora de maíz. Obtenido de Perfeccionadora en espiga desgranadora de maíz: http://patentados.com/ invento/perfeccionamientos-maquinas-trilladorasdeshojadoras-desgranadoras-maiz.2.html

allbis. (07 de Setiembre de 2017). Motores y Maquinarias. El Salvador. Obtenido de https: / / antiguo-cuscatlan-li.all.biz/desgranadora-de-maiz-g78\#. WbIefevhDIU

Azteca. (08 de Setiembre de 2017). Obtenido de https://www.engormix.com/molinos-azteca-juper/desgranadoras-picadoras-maiz-forraje-azteca-sh4112_pr26241.htm
CEPAL, FAO, IICA. (2014). Perspectivas de la agricultura y del desarrollo rural en las américas: una mirada hacia América Latina y el Caribe. San José C.R.: IICA.

Colorado State University, Health professions advising. (11 marzo 2004). http://cls.casa.colostate. edu/ cultivostransgenicos/sp_hotstarlink.html.

Cruz, P., Romantchick, E., Hahn, F. (2010). Diseño, construcción y evaluación de una máquina para deshojar la mazorca de maíz para la envoltura de tamal. Ingeniería agrícola y Biosistemas, 69 - 74.

Curiosfera. (03 de mayo de 2017). ¿Qué es el maíz? Obtenido de http://www.curiosfera.com/que-es-elmaiz-historia-expansion-consumo/

De Los Ríos, J. (2008). La agricultura latinoamericana y sus posibilidades de adapatación a los cambios climáticos globales. IDeAS, 2(1), 53-75.

Donoso, J. (2007). Situación del sector de maquinaria agrícola en América Latina. Rosario: STRAT Consulting.

Enac, J. y Enac L. (1970.). España. Patente no 384194.

FAO, T. (205). Producción artesanal de semilla de maíz para el pequeño agricultor. Tecnologías y prácticas para pequeños productores agrarios, 2.

FAO. (2015). El maíz en los trópicos: Mejoramiento y producción. Obtenido de http://www.fao.org/docrep/003/x7650s/x7650s24.htm

Hall, A., Hollowenko, A., \& Laughlin, H. (1984). Diseño de Maquinas. México: Mc Graw Hill.

Hernández, J. (enero de 2009). El origen y la diversidad del maíz en el continente americano. Obtenido de Greenpeace: http://www.greenpeace.org/mexico/ global/mexico/report/2009/3/el-origen-yla-diversidad-del.pdf

Inurritegui, M., Camacho, I., Ludeña, J. (2008). Acuerdo Asociación UE-CAN: ¿Quiénes ganan y quiénes pierden? Un estudio exploratorio sobre el sector agrícola. Lima: Centro Peruano de Estudios Sociales.

Maíces del Nansa. (1 de octubre de 2013). Algunas formas tradicionales del desgranado. Manual de maíz. Obtenido de: Algunas formas tradicionales del desgranado. Manual de Maíz: http://maicesdelnansa. blogspot.com/2013/10/algunas-formas-tradicionalesdel.html

Ministerio de Agricultura, Ganadería, acuacultura y pesca. (2014). Maíz duro seco. Obtenido de http:// sinagap.agricultura.gob.ec/phocadownloadpap/ BoletinesCultivos/maizd uro.pdf 
Monteros A., Salvador S. (2015). Rendimientos de maíz duro seco en el Ecuador invierno. SINAGAP

Moya, C. (2014). Diseño y construcción de una máquina deshojadora de maíz seco que, partiendo de la mazorca con su envoltura, entregará como producto la mazorca y como subproducto, la envoltura del maíz. Sangolquí.

Nansa, F. (2013). Formas tradicionales del desgranado manual de maíz. Maíces de la Nansa, 1; 2.

Obrador Rousseau, J. (2014). Cosecha de granos. Chile: INIA-Estación Experimental. La Platina.

Observatorio Regional de innovaciones tecnológicas en cadenas de maíz y frijol. (2013). Guía para el trillado de frijol y desgranado de maíz. Obtenido de Guía para el trillado de frijol y desgranado de maíz: http:// www.observatorioredsicta.info/es/publicaciones / guia / 2013 / guia-parael-trillado-de-frijo-y-desgranado-de-maiz

Ozaeta, A., Flórez, L., \& Higuera, O. (17 de noviembre de 2013). Scientific Electronic Library Online. Obtenido de http://www.scielo.org.co/ scielo.php?pid =S0121-11292013000200004\&scr ipt $=$ sci_arttext

Phal, G., \& Beitz, W. (1996). Engineering Design. London: Springer.

Rodríguez, H. (s.f.). Ingemecánica. Obtenido de http:/ /ingemecanica.com/tutorialsemanal/tutorialn121.html 92

SKF Rodamientos. (15 de junio de 2015). SFK Rodamientos. Obtenido de SFK Rodamientos: http://www.skf.com/co/products/ bearings-unitshousings / bearing-units / ballbearing-units/y-bearing-flanged-units / casthousing-square-flange-grub.screwlocking/index.html? prodid $=2125138108 \&$ imperial $=$ true

SKF. (2014). SKF Power Transmission products. Obtenido dehttp://www.skf.com/binary/21- 139508/ Power_Transmission_Products_11015_EN.pdf

Soler y Palau. (2011). Catálogo Sy P. Obtenido de http: / / www.fmcm.com.mx/manuales/Soler\&Palau/ Comercial\%20e\%20Industrial.pdf

SoloStocks. (30 de agosto de 2017). Obtenido de http://www.solostocks.com/venta-productos / otros/desgranadora-manual-de-maiz-econ-17870150

Torotrac. (01 de setiembre de 2017). Obtenido de http: / / www.torotrac.com/product/OE-Desgr anadora-1.
Ulrich, K., Eppinger, S. (2013). Diseño y desarrollo de productos (Quinta ed.). México: Alfaomega.

Ureña J., Villavicencio J. (2012). Diseño y construcción de una máquina para el proceso de desgranado de maíz de la Costa, Riobamba.

Weiku. (2012). Venta de máquinas agrícolas. Obtenido de Venta de máquinas agrícolas: http: / /www.wei$\mathrm{ku}$.com/products/19706663/Sale_home_use_ wax_candle_making_machine_candle_extruder_ machine_Mobile_0086_15238020768.html

Yánez Camacho, F. M. (2010). Estudio del sistema mecánico aplicado al desgranado de maíz suave seco para mejorar tiempos de producción en el cantón San Miguel provincia de Bolivar. Obtenido de Estudio del sistema mecánico aplicado al desgranado de maíz suave seco para mejorar tiempos de producción en el cantón San Miguel provincia de Bolívar: http://repositorio.uta. edu.ec/bitstream/123456789/1267/1/Tesis\%20 I. \%20M.\%20112\%2020Y\%C3\%A 1 nez\%20 Camacho\%20Fredy\%20Miguel.pdf 